\title{
Análisis del comportamiento sísmico de los edificios de obra de fábrica, típicos del distrito Eixample de Barcelona
}

\author{
Seismic performance analysis of the masonry buildings, typical \\ of the Eixample district of Barcelona
}

R. Moreno-González ${ }^{(*)}$, J. M. Bairán ${ }^{(*)}$

RESUMEN

En este trabajo se realiza un estudio para evaluar la vulnerabilidad y el riesgo sísmico de los edificios de obra de fábrica de ladrillo ampliamente existentes en el distrito Eixample de Barcelona, España. Los edificios analizados son reales y se dispone de planos e informes específicos. El análisis del riesgo sísmico se lleva a cabo mediante un método mecánico basado en el Método del Espectro de Capacidad. La demanda sísmica se define a partir del espectro elástico de respuesta definido por el Eurocódigo-8. Las curvas de fragilidad se obtienen a partir de un análisis no lineal, teniendo en cuenta los espectros de capacidad. El daño sísmico esperado se consigue con las matrices de probabilidad de daño. La modelización de los edificios se realiza con el programa TreMuri mediante un modelo de macroelementos. Los resultados obtenidos muestran una vulnerabilidad importante, teniendo en cuenta el escenario sísmico considerado, el riesgo sísmico esperado es alto.

\section{SUMMARY}

In this work a study about the vulnerability and seismic risk of the unreinforced masonry buildings, widely, existent in the Eixample district of Barcelona, Spain, is carried out. The buildings, here, analyzed are real and detailed structural drawings and reports have been used to model them. The seismic risk is evaluated by means of a mechanical model, based in the Capacity Spectrum Method. The seismic demand is described by elastic response spectrum defined by the Eurocode-8. Fragility curves are obtained from a nonlinear analysis, considering the capacity spectra. Expected seismic damage is gotten with the damage probability matrices. The analysis of the buildings has been performed by TreMuri program by means of a macro-element model. The results shown a considerable vulnerability in this type of buildings, therefore, in spite of the seismic hazard, the expected seismic risk is significant.

409-13

Palabras clave: mampostería, vulnerabilidad, fragilidad, capacidad, daño sísmico.
Keywords: masonry, vulnerability, fragility, capacity, seismic damage. 


\section{INTRODUCCIÓN}

El comportamiento sísmico inadecuado de las estructuras es la causa principal de pérdidas humanas y económicas, básicamente, los avances que se realizan en el diseño de estructuras se aplican a las estructuras nuevas y, en menor medida, a la rehabilitación de estructuras existentes, a pesar de que el número de edificios existentes es mucho mayor que las estructuras nuevas. En zonas de sismicidad moderada o baja es frecuente encontrar que las construcciones no incluyan ningún tipo de análisis o diseño antisísmico, debido a que las normativas no suelen obligar a ello o que no existían en el momento de su construcción o, sencillamente, que la población no reconoce el evento sísmico como un factor de riesgo para su comunidad.

Para poder reducir el número de pérdidas humanas y económicas hay que comenzar por realizar estudios de vulnerabilidad sísmica y alertar a las autoridades gubernamentales para que tomen medidas de precaución y seguridad sobre la amenaza sísmica existente.

No existe una metodología estándar para estimar la vulnerabilidad sísmica de los edificios, ni de las estructuras en general. La base fundamental para estudiar la vulnerabilidad o el riesgo sísmico, a nivel estructural, es disponer de una amplia información sobre los elementos con los que se pretende trabajar para evaluar la vulnerabilidad sísmica y, a partir de aquí, calcular el daño probable que se pueda sufrir a causa de un sismo.

Tabla 1

Distribución de las tipologías existentes en Barcelona

\begin{tabular}{|c|c|c|}
\hline Tipologías & Descripción & $\begin{array}{l}\text { \% Edificios } \\
\text { existentes }\end{array}$ \\
\hline M31 & $\begin{array}{l}\text { Edificio de obra de fábrica con forjado de } \\
\text { viguetas de madera. }\end{array}$ & 32 \\
\hline M32 & $\begin{array}{l}\text { Edificio de obra de fábrica con forjado de } \\
\text { bóveda de revoltón cerámico. }\end{array}$ & 18 \\
\hline M33 & $\begin{array}{l}\text { Edificio de obra de fábrica con forjado de } \\
\text { viguetas metálicas y bóveda de revoltón ce- } \\
\text { rámico. }\end{array}$ & 26 \\
\hline M34 & $\begin{array}{l}\text { Edificio de obra de fábrica con forjado de } \\
\text { viguetas de hormigón y bóveda de revoltón } \\
\text { cerámico. }\end{array}$ & 1 \\
\hline $\mathrm{RC} 32$ & $\begin{array}{l}\text { Edificio de hormigón armado con forjado reti- } \\
\text { cular, sistema estructural irregular y paredes de } \\
\text { relleno dispuestas de forma irregular. }\end{array}$ & 18 \\
\hline S1 & Edificio metálico porticado. & 1 \\
\hline S2 & Edificio metálico arriostrado. & 1 \\
\hline S3 & $\begin{array}{l}\text { Edificio metálico de pórticos con paredes de } \\
\text { obra de fábrica. }\end{array}$ & 1 \\
\hline S5 & $\begin{array}{l}\text { Edificio metálico porticado con sistemas for- } \\
\text { mados de hormigón (forjado de hormigón). }\end{array}$ & 2 \\
\hline
\end{tabular}

En este trabajo se presenta un estudio corespondiente a la vulnerabilidad y el riesgo sísmico de los edificios de obra de fábrica de ladrillo existentes en el distrito Ensanche ("Eixample" en catalán) de Barcelona, España. Para llevar a cabo este estudio se emplea una metodología que contempla la modelización de edificios para evaluar el daño sísmico mediante técnicas basadas en el desempeño sísmico.

La Municipalidad de Barcelona tiene una base catastral, la cual está muy bien documentada en el informe Infocca (1), referente a la edad y características constructivas de los edificios; permite determinar las características geométricas, en planta y en altura de los edificios de cada parcela. Se dispone de información de 63.000 edificios que representan, aproximadamente, el $91 \%$ del total de edificios, los cuales corresponden a edificios residenciales. De estos edificios se conoce su configuración en planta y en altura, su situación dentro de la manzana, si forman parte de un sistema de agregado o no de edificios; la tipología constructiva, el año de construcción y el estado de conservación; además, se han conseguido algunos planos arquitectónicos y estructurales de varios edificios existentes, así como también, informes de patologías.

Casi la mitad de los edificios residenciales existentes en Barcelona han sido construidos antes del año 1940, siendo 1931 el año medio de construcción de los edificios del Eixample. A finales del siglo XIX y principios del siglo $X X$, el sistema constructivo más frecuente, en Barcelona, utilizaba muros de carga de obra de fábrica de ladrillo y forjados de bovedilla con vigas de madera o viguetas metálicas. En el distrito Eixample, aproximadamente, el $75 \%$ de los edificios pertenecen a esta tipología.

En general, la tipología que representa un mayor número de edificios son los edificios de obra de fábrica de ladrillo, seguido por, los edificios de hormigón armado con forjados reticulares y, finalmente, los edificios metálicos, estos últimos representan un $5 \%$ de los edificios existentes. La Tabla 1 muestra la distribución de los edificios existentes, en la ciudad de Barcelona, según su tipología (2).

El objetivo principal de este trabajo es obtener las matrices de probabilidad de daño para evaluar el daño sísmico esperado de los edificios de obra de fábrica existentes en el Eixample de Barcelona. El análisis se ha llevado a cabo mediante una metodología basada en el método del espectro de capacidad, la cual ha sido desarrollada dentro del marco del proyecto europeo Risk-UE $(2,3)$. Para estudiar el daño sísmico esperado en este tipo de edificaciones se ha considerado un 
escenario sísmico definido por la norma sísmica española NCSE-02 (4) y el Eurocódigo 8 (5), donde la aceleración sísmica básica para Barcelona es de 0,04 g. Después de definir las propiedades mecánicas y geométricas y los materiales, se evalúa la capacidad de los edificios mediante el programa TreMuri (6), este permite realizar análisis estático y dinámico no lineal de edificios de mampostería, en 3D, obteniendo las curvas de capacidad y los espectros de capacidad bilineales para cada edificio, seguidamente, se definen las correspondientes curvas de fragilidad. A partir de aquí se obtienen las matrices de probabilidad de daño.

\section{LA CIUDAD DE BARCELONA}

En Barcelona, durante la primera mitad del siglo XIX se intensifica la urbanización del interior de la ciudad que, progresivamente, se dota del aspecto e infraestructuras de una ciudad moderna. Entre 1858 y 1868 se derriban las murallas de la ciudad y se inicia el gran proyecto urbanístico para la construcción de la Barcelona moderna, una nueva ciudad abierta e industrial. Lo que da paso al nacimiento de la Barcelona actual es el distrito l'Eixample, el cual fue proyectado por el ingeniero Ildefonso Cerdá (7). Este proyecto de expansión de la ciudad permitió unir el núcleo de la ciudad con los pueblos que darán nombre a los distritos y barrios de la ciudad actual. La aprobación del "Plan del Eixample" de Barcelona, de Ildefonso Cerdá, en el año 1859 va a cambiar y a convertir a la ciudad catalana en un referente del urbanismo moderno. Este plan urbanístico tiene ya 150 años y ha permitido a la ciudad crecer ordenadamente y resolver con eficiencia los problemas de una ciudad atrapada dentro de murallas; también ha significado la unión armónica entre historia y progreso.

El censo de 1900 establece en 553.000 el número de habitantes de esta ciudad ampliada. Entre 1909 y 1929 Barcelona experimenta una colosal expansión, de los 587.411 habitantes de 1910 se pasa a 1.005 .565 en 1930. Entre 1940 y 1960 se produce un considerable crecimiento sostenido y la ciudad gana medio millón de habitantes. Este incremento de la población es debido, principalmente, al aumento de la natalidad y al gran número de inmigrantes procedentes del Sur de España. A pesar de que la Carta Municipal de 1961 otorga mayor autonomía a la ciudad, durante la alcaldía de Porcioles (1957-1973) se produjo un crecimiento especulativo y desordenado con grandes deficiencias estructurales. A partir de 1978 se inicia un mayor control de la sociedad sobre las instituciones y el gobierno de la ciudad. En la actualidad, Barcelona está delimitada entre las montañas de Montjuïc

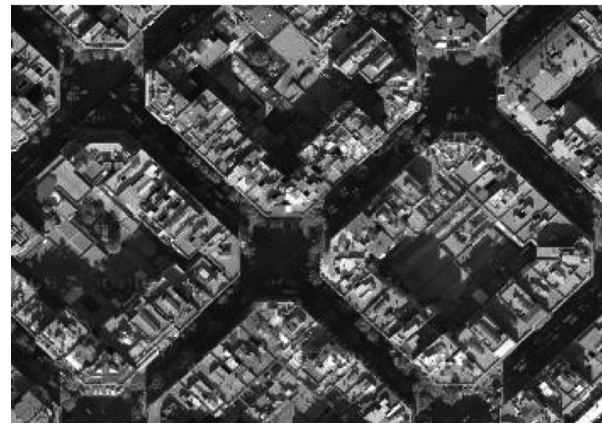

y Collserola y el río Llobregat y el río Besós, que limitan claramente su crecimiento en superficie, dejando, como única alternativa de crecimiento, la reconstrucción de zonas en desuso o industriales. Los últimos ejemplos de transformación urbanística de la ciudad son la Villa Olímpica en 1992 y la apertura al mar de la Avinguda Diagonal con el Forum Universal de les Cultures en el 2004. El distrito del Eixample destaca como la principal área de Barcelona donde se acumula una importante población, una notable actividad económica y un importante patrimonio cultural $(8,9)$. Actualmente, el municipio de Barcelona se extiende en una superficie de 9.907 Ha con una población de 1,5 millones de habitantes según el censo de 2001.

\subsection{El sistema constructivo}

El Ensanche de Barcelona está formado por bloques o manzanas simétricas de $113 \times 113$ $\mathrm{m}$, perfectamente alineadas, y con chaflanes de una longitud de $20 \mathrm{~m}$. En cada manzana o bloque hay un promedio de 25 edificios, en su mayoría son edificios de obra de fábrica de ladrillo (edificios de mampostería), aunque es frecuente encontrar que entre dos edificios viejos se haya construido uno nuevo de hormigón armado con forjados reticulares u ocasionalmente, metálico. En la Figura 1 se muestra una vista típica de las manzanas que forman el distrito.

Los edificios son de paredes de carga, los cuales muestran esquemas muy repetitivos, tales como: cimentación superficial sobre zapatas corridas bajo muros, o aisladas bajo pilares, los elementos resistentes son muros de carga y elementos de sustentación en planta baja, sobre un sistema de jácenas metálicas o de pórticos de hormigón se apoyan las paredes de carga o bien secundarias, normalmente las jácenas metálicas se apoyan sobre paredes de mampostería.

Los forjados son unidireccionales y presentan luces cortas, es previsible que se trate de forjados, unidireccionales, de viguetas de madera, metálicas, o de hormigón sin capa superior de compresión. 
Principalmente, estos edificios presentan una geometría rectangular y en la parte central de los edificios suele existir uno o más núcleos parcialmente cerrados mediante paredes de fábrica de ladrillo de $10 \mathrm{~cm}$ de espesor, formados alrededor de las cajas de escaleras o de los patios de luces. La distribución de las paredes de carga responde a tres esquemas estructurales fundamentales (10):

- Los muros de fachada (calle y patio de manzana) son de $30 \mathrm{~cm}$ de espesor y actúan como paredes de carga (muros portantes) junto a un conjunto de paredes interiores de carga, paralelas a aquéllas, de $15 \mathrm{~cm}$ de espesor.

- En el caso de edificios estrechos, el forjado se forma en sentido transversal actuando las paredes de medianería (secundarias) como muros de carga.

- En el caso de edificios de esquina, la distribución es en forma trapecial, las fachadas de calle y patio actúan como paredes de carga, se dispone de una segunda pared de carga interior paralela a la fachada de calle y se utilizan otras paredes interiores como elementos de carga. De esta forma, existen paredes de carga en, al menos, tres direcciones, separadas angularmente a $45^{\circ}$ (una dirección transversal, paralela al chaflán, y dos direcciones diagonales al eje y paralelas a los lados de la manzana).

Normalmente, las paredes alcanzan longitudes importantes, $15 \mathrm{~m}$. En las paredes existen aberturas para puertas y ventanas que dan lugar a dinteles o antepechos de anchura y altura variables. Según el año de construcción, los forjados pueden estar formados de viguetas de madera, metálicas o de hormigón. Los forjados de vigueta metálica y revoltón cerámico son comunes en los techos de planta baja, apoyándose en jácenas y pilares metálicos o bien en paredes y los forjados de vigueta de madera y revoltón cerámico son comunes en el resto de las plantas, están formados por dos hojas de rasilla y sobre éstas existen cascotes en relleno de senos, mortero de cal y pavimento. El revoltón constituye el sistema tradicional y las bovedillas de cerámica o de mortero son rellenas de cascotes de cemento o mortero de baja resistencia.

Los edificios del Eixample se pueden describir en cuatro épocas o períodos:

-Pre-modernismo: 1860-1900

-Modernismo: 1890-1910

-Post-modernismo: 1910-1936, siendo el sistema constructivo predominante la mampostería

- El último período se da a partir de la década de los 60, cuando el hormigón armado como sistema constructivo en edificaciones empieza a ser importante, dando lugar al comienzo de la arquitectura contemporánea (4).
La mayoría de los edificios de obra de fábrica o mampostería tienen de 6 a 8 pisos y presentan alturas diferentes en las primeras plantas respecto al resto de los pisos. Hoy en día, estas primeras plantas son dedicadas al comercio.

\section{DESCRIPCIÓN Y MODELIZACIÓN DE LOS EDIFICIOS}

En el sistema constructivo del Eixample se pueden distinguir dos tipos de edificios, los edificios de línea o banda y los edificios de esquina o chaflán. Estos edificios, con las mismas proporciones dan como resultado unas técnicas constructivas que se sistematizan debido a que se repiten constantemente, dando origen a un sistema constructivo que varía básicamente en la fachada. En este apartado se definen los tres edificios seleccionados para representar los edificios de obra de fábrica de ladrillo ampliamente existentes en Barcelona y, además, se utilizan para evaluar la vulnerabilidad de los edificios.

Los edificios Ilamados Casti y Pelai son representativos de los edificios situados dentro de las hileras de edificios, cuya fachada principal da a una misma calle. Estos edificios, de planta rectangular, son los Ilamados edificios de banda, es decir, no forman esquina. El edificio Ilamado Angolo tiene una planta más irregular $y$, muestra tres secciones de fachada, dos que dan a calles distintas (calles perpendiculares) y una en chaflán que las une. Este edificio Angolo es típico de las esquinas correspondientes a los cruces entre calles.

La Figura 2 muestra las plantas de estos edificios con la numeración de las paredes. Los tres edificios son reales y típicos en I'Eixample, las plantas han sido tomadas directamente de planos originales. En la Tabla 2 se especifican las características en planta y altura de los edificios estudiados.

En cuanto al edificio Casti, las paredes de la fachada principal y trasera tienen espesores de $50 \mathrm{~cm}$ y $45 \mathrm{~cm}$, respectivamente, reduciéndose a partir del primer piso unos $10 \mathrm{~cm}$ y $15 \mathrm{~cm}$, respectivamente. Las paredes laterales o medianeras tienen un espesor de $30 \mathrm{~cm}$ en la planta baja y de $15 \mathrm{~cm}$ en el resto de los pisos, existen otras paredes interiores de $15 \mathrm{~cm}$ de espesor.

En el edificio Pelai, las paredes son de 15 $\mathrm{cm}$ de espesor y las paredes de fachadas de $30 \mathrm{~cm}$. En las dos primeras plantas existen pilares y jácenas metálicas, los cuales soportan el peso de las paredes superiores.

En cuanto al edificio Angolo, presenta una mayor diversidad de formas geométricas, las paredes están dispuestas de forma paralela las unas con las otras para crear un sistema resistente. El muro de fachada de calle tiene 
un espesor de $40 \mathrm{~cm}$ y el de la fachada trasera de $30 \mathrm{~cm}$, las paredes interiores de carga son de $15 \mathrm{~cm}$ de espesor. El sistema resistente interior en las dos primeras plantas está formado de pilares y jácenas metálicas.

Para todos los edificios se ha considerado el mismo tipo de forjado, es un forjado de losas de viguetas de madera, cubiertas de revoltón y con una base de baldosa doblada encajada en el interior para sostenerlo $y$, finalmente, cubierto de mortero de cal, Figura 3, este tipo de forjado junto a los de viguetas metálicas son los más utilizados.

Para el estudio aquí realizado, se analizó cada edificio con el programa TreMuri (6). El programa TreMuri es un programa tridimensional de análisis no lineal para estructuras de mampostería y ha sido desarrollado en la Universitat degli Studi di Genova, Italia (6).

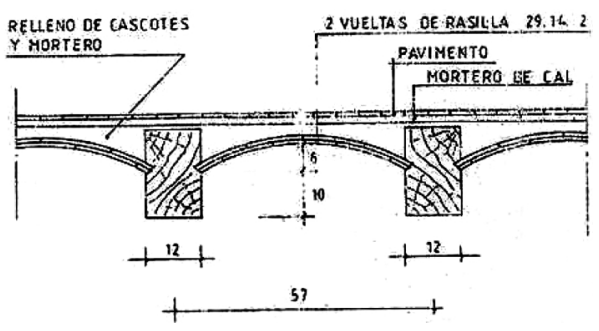

\subsection{Programa TreMuri}

El programa TreMuri es un programa tridimensional de análisis no lineal para estructuras de fábrica de ladrillo. El programa representa los paneles de mampostería mediante un modelo no lineal de macroelementos propuesto por Gambarotta y Lagomarsino (11). El TreMuri es un programa de macroelementos, proyectado para un análisis incremental no lineal con control de fuerza o desplazamiento, análisis estático, análisis modal y análisis dinámico con excitación sísmica en la base de la estructura, bidimensional o tridimensional.

El modelo de daño del macromodelo utilizado en el programa para ensamblar el modelo de la pared, tiene en cuenta el efecto de la resistencia a compresión limitada de la mampostería, especialmente en el mecanismo del vuelco (12). El modelo incluye los efectos de agrietamiento por medio de una ley constitutiva no lineal con deterioro de la rigidez en compresión (13).

Aparte de sus características geométricas, el macroelemento es definido por seis parámetros: el módulo de cortante, la rigidez axial, la resistencia a cortante de la mampostería, un coeficiente adimensional que controla la deformación inelástica, un coeficiente global de fricción y un factor que controla la fase del ablandamiento (13).
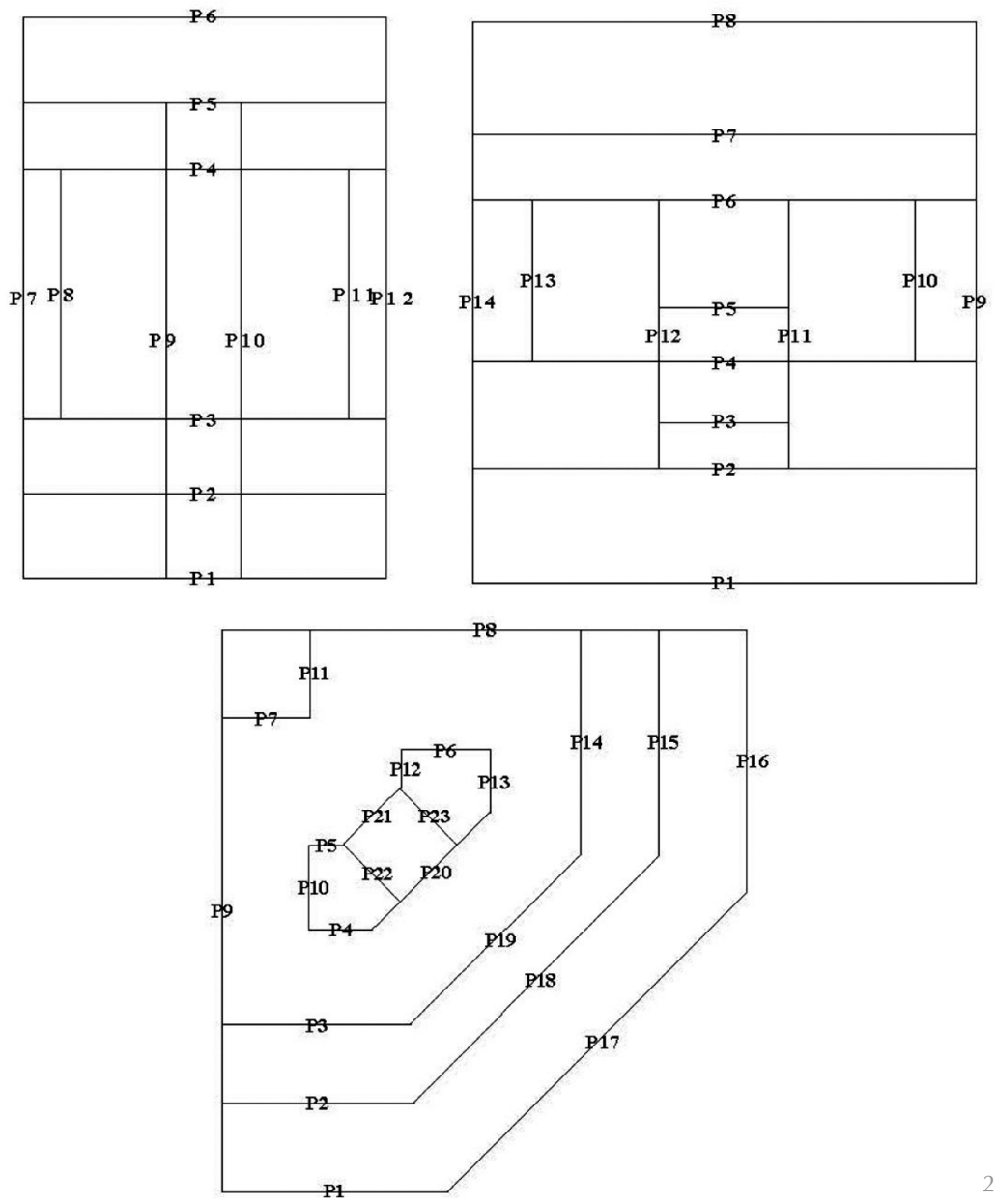

Tabla 2

Características de los edificios analizados

\begin{tabular}{|c|c|c|c|}
\hline Edificios & CASTI & PELAI & ANGOLO \\
\hline Año de construcción & $1930-1931$ & $1882-1886$ & 1936 \\
\hline $\mathrm{H}(\mathrm{m})$ & 22,0 & 24,4 & 22,4 \\
\hline Dimensiones $(\mathrm{m})$ & $12,65 \times 27,00$ & $18,40 \times 23,70$ & $\mathrm{~A}=557 \mathrm{~m}^{2}$ \\
& $\mathrm{~A}=341,55 \mathrm{~m}^{2}$ & $\mathrm{~A}=436,08 \mathrm{~m}^{2}$ & $\mathrm{P}=94,30 \mathrm{~m}$ \\
\hline $\mathrm{N}^{\text {o }}$ pisos & 7 & 6 & 8 \\
\hline h. planta baja $(\mathrm{m})$ & 4,0 & 4,8 & 3,1 \\
\hline h. 1 $^{\text {er }}$ piso $(\mathrm{m})$ & 3,0 & 4,0 & 2,5 \\
\hline h. resto pisos $(\mathrm{m})$ & 3,0 & 3,9 & 2,8 \\
\hline
\end{tabular}

Los modelos numéricos que incorpora el programa han sido calibrados cuidadosamente, de forma que el programa es capaz de reproducir los mecanismos de disipación observados en casos reales. En consecuencia, TreMuri es capaz de describir y simular el comportamiento no lineal en el plano, de los paneles de mampostería y permite estimar el daño que este tipo de edificios sufren, cuando se ven sometidos a sismos.

Las Figuras 4, 5 y 6 muestran los 3 edificios modelados con el programa TreMuri, en ellas se muestra el modelo 3D y una de sus paredes. Antes de llevar a cabo el análisis es necesario definir las propiedades mecánicas de los materiales.
2. Plantas de los edificios Casti (arriba izquierda), Pelai (arriba derecha) y Angolo (abajo).

3. Forjado de madera típico de Eixample, (tomado de los planos). 
4. Vista 3D del edificio Casti, modelado con el programa TreMuri.

5. Vista 3D del edificio Pelai, modelado con el programa TreMuri.

6. Vista 3D del edificio Angolo, modelado con el programa TreMuri.

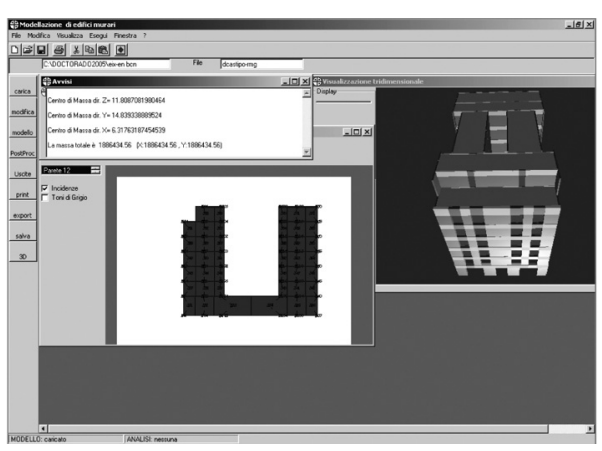

y muros de mampostería. La misma Tabla 3 muestra los parámetros para las configuraciones constructivas aquí definidas, las características de los forjados, las propiedades de los pilares y jácenas metálicas.

\section{METODOLOGÍA}

Lo más reciente en el campo de la evaluación de la vulnerabilidad y el riesgo sísmico se basa en modelos mecánicos. En este trabajo se sigue un procedimiento simplificado propuesto en el proyecto RiskUE (2) y basado en el método del espectro de capacidad.

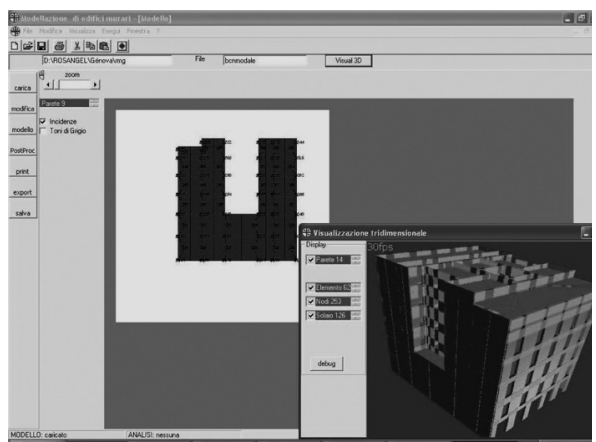

La vulnerabilidad de un edificio existente se puede entender como la capacidad que tiene la estructura para soportar acciones sísmicas y la fragilidad está relacionada con su vulnerabilidad sísmica y se cuantifica mediante curvas de fragilidad.

La acción sísmica se define por medio del espectro elástico de respuesta con $5 \%$ de amortiguamiento y está representado en coordenadas espectrales (aceleración espectral, en el eje de las ordenadas, y desplazamiento espectral, en las abscisas).

Teniendo en cuenta los modelos de los edificios existentes y las técnicas constructivas es posible construir las curvas de capacidad a partir de un análisis estático no lineal. Las curvas de capacidad definen la resistencia estructural del edificio y, gráficamente, se representan con el cortante en la base, eje de las ordenadas, y el desplazamiento lateral del último piso, eje de las abscisas.

\subsection{Características mecánicas}

Para evaluar el comportamiento de los edificios es necesario conocer sus propiedades mecánicas. Se desconoce la existencia de estudios rigurosos que permitan definir con exactitud las propiedades mecánicas de estos materiales. En base a las especificaciones técnicas al uso, a las características constructivas, a los materiales utilizados, a estudios patológicos realizados en algunos edificios, a estudios realizados por otros investigadores (14) y, junto con la ayuda de la opinión de ingenieros con experiencia en el conocimiento del comportamiento mecánico de la obra de fábrica de ladrillo sin armar, se ha realizado un volumen importante de ensayos numéricos con el programa TreMuri, lo que ha permitido definir los parámetros mecánicos que requiere la definición de los macroelementos.

La Tabla 3 contiene los parámetros característicos de los macroelementos tipo pilar y tipo dintel, utilizados para modelar paredes
Las curvas de capacidad se pueden representar en coordenadas espectrales a partir de las propiedades modales, de esta manera, se tiene, en un mismo sistema de coordenadas, la capacidad estructural y la demanda sísmica. Esta nueva representación recibe el nombre de espectro de capacidad.

Con la finalidad de tener parámetros objetivos y cuantificables para evaluar la capacidad y fragilidad de una estructura, el espectro de capacidad se representa por medio de una curva de capacidad bilineal simplificada, definida por dos puntos de control: capacidad de cedencia (Dy, Ay) y capacidad última (Du, Au), Figura 7. El punto de capacidad de cedencia representa el desplazamiento en el que la respuesta del edificio empieza a ser no lineal y el punto de capacidad última representa el desplazamiento en el que el sistema estructural ha alcanzado el mecanismo de colapso o su máxima capacidad (15). Los umbrales de los estados de daño representados en la Figura 7 se definen en la Tabla 4. 
La curva de capacidad bilineal se determina mediante un criterio de balance de energía, de esta manera se busca que la curva bilineal pueda absorber la misma energía que la curva de capacidad real y que tenga el mismo punto de desplazamiento último.

El espectro de demanda es la representación de la acción sísmica y se basa en el espectro de respuesta de diseño de la zona de estudio, con 5\% de amortiguamiento, reducido para niveles mayores de amortiguamiento efectivo.

El punto clave para la estimación del daño esperado de un edificio sometido a una acción sísmica es la determinación del máximo desplazamiento espectral que este va a experimentar. Esta respuesta máxima se conoce como punto de desempeño o punto de capacidad por demanda.

El espectro de demanda y el punto de desempeño son obtenidos a partir del espectro de respuesta y del espectro de capacidad mediante un proceso iterativo definido en el ATC-40, procedimiento A (16). El punto de desempeño se obtiene en el punto de intersección del espectro de capacidad y el espectro de demanda, Figura 8.

Las curvas de fragilidad definen la probabilidad de alcanzar o exceder un estado de daño límite específico, dada una respuesta estructural, ante una acción sísmica dada. Las curvas de fragilidad siguen una distribución de probabilidad lognormal y se construyen con respecto a una variable que caracteriza la acción sísmica, en este caso, el desplazamiento espectral. Así pues, las curvas de fragilidad se definen como: [1]

$$
P\left[E D \geq E D_{i}\right]=\Phi\left[\frac{1}{\beta_{E D}} \cdot \ln \left(\frac{S d}{\overline{S d}_{E D}}\right)\right]
$$

Donde $S d$ ED es el desplazamiento espectral medio para el cual la probabilidad de excedencia es del $50 \%, \beta_{E D}$ es la desviación estándar del logaritmo natural del desplazamiento espectral para el estado límite de daño, en otras palabras, $\beta_{E D}$ es la variabilidad asociada al estado de daño, $\Phi$ es la función de distribución normal estándar acumulada, $S d$ es el desplazamiento espectral; $E D$ indica el estado de daño y viene definido como: 1-para el estado de daño leve, 2-para moderado, 3-para severo y 4-el estado de daño completo (justo antes de que se produzca el colapso). Gráficamente se representa, en las ordenadas, con la $P\left(E D>E D_{i}\right)$ y el desplazamiento espectral $(S d)$, en las abscisas.

Las curvas de fragilidad pueden ser obtenidas de forma simplificada a partir de la curva de capacidad bilineal.

\begin{tabular}{|c|c|c|}
\hline \multicolumn{3}{|c|}{$\begin{array}{c}\text { Tabla } 3 \\
\text { Propiedades mecánicas de los elementos } \\
\text { Paredes de Mampostería }\end{array}$} \\
\hline Módulo elástico, E & \multicolumn{2}{|c|}{$1.800 \mathrm{MPa}$} \\
\hline Módulo a cortante, G & \multicolumn{2}{|c|}{$300 \mathrm{MPa}$} \\
\hline Resistencia a cortante, $\tau_{k}$ & \multicolumn{2}{|c|}{$0,12 \mathrm{MPa}$} \\
\hline Resistencia a compresión, $f_{m k}$ & \multicolumn{2}{|c|}{ 4,0 MPa } \\
\hline Peso específico, $\gamma$ & \multicolumn{2}{|c|}{$1.800 \mathrm{~kg} / \mathrm{m}^{3}$} \\
\hline Parámetro de ablandamiento para pilares, $\beta_{p}$ & \multicolumn{2}{|c|}{0,20} \\
\hline Parámetro de ablandamiento para vigas, $\beta_{d}$ & \multicolumn{2}{|c|}{0,05} \\
\hline \multicolumn{3}{|c|}{ Forjado de Madera } \\
\hline Módulo elástico, $E_{1}$ & \multicolumn{2}{|c|}{$4.000 \mathrm{MPa}$} \\
\hline Módulo elástico, $E_{2}$ & \multicolumn{2}{|c|}{$40 \mathrm{MPa}$} \\
\hline Módulo a cortante, $G$ & \multicolumn{2}{|c|}{$100 \mathrm{MPa}$} \\
\hline Carga, $q$ & \multicolumn{2}{|c|}{$500 \mathrm{~kg} / \mathrm{m}^{2}$} \\
\hline \multicolumn{3}{|c|}{ Perfiles Metálicos } \\
\hline & Pilares & Vigas \\
\hline Tipo de perfiles & HEB-200 & IPN-360 \\
\hline Módulo elástico, $E_{s}$ & 2,1E5 MPa & 2,1E5 MPa \\
\hline Área, $A$ & $0,00781 \mathrm{~m}^{2}$ & $0,0097 \mathrm{~m}^{2}$ \\
\hline Momento de inercia, $I_{y}$ & $56,97 \mathrm{E}-6 \mathrm{~m}^{4}$ & $19,61 \mathrm{E}-5 \mathrm{~m}^{4}$ \\
\hline Peso específico, $\gamma$ & $7.850 \mathrm{~kg} / \mathrm{m}^{3}$ & $7.850 \mathrm{~kg} / \mathrm{m}^{3}$ \\
\hline
\end{tabular}

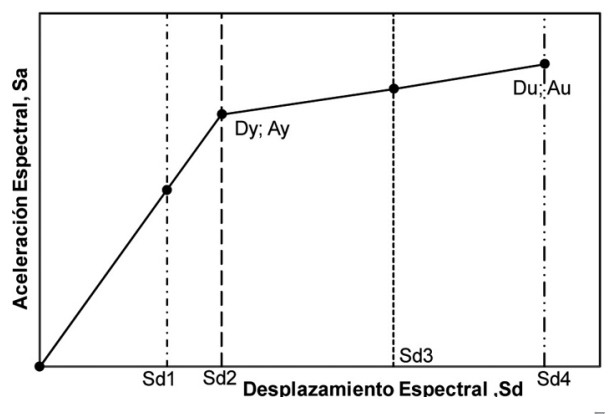

Tabla 4

Definición de los umbrales

de los estados de daño

\begin{tabular}{|c|c|}
\hline $\begin{array}{c}\text { Umbrales } \\
\text { de daño }\end{array}$ & Definición \\
\hline Leve & ${\overline{S d_{1}}}_{1}=0,7 \cdot D y$ \\
\hline Moderado & $\overline{S d}_{2}=D y$ \\
\hline Severo & $S \overline{S d}_{3}=D y+0,25 \cdot(D u-D y)$ \\
\hline Completo & $\overline{S d}_{4}=D u$ \\
\hline
\end{tabular}

7. Definición del espectro de capacidad bilineal y de los umbrales de los estados de daño.

8. Definición del espectro de demanda y punto de desempeño.

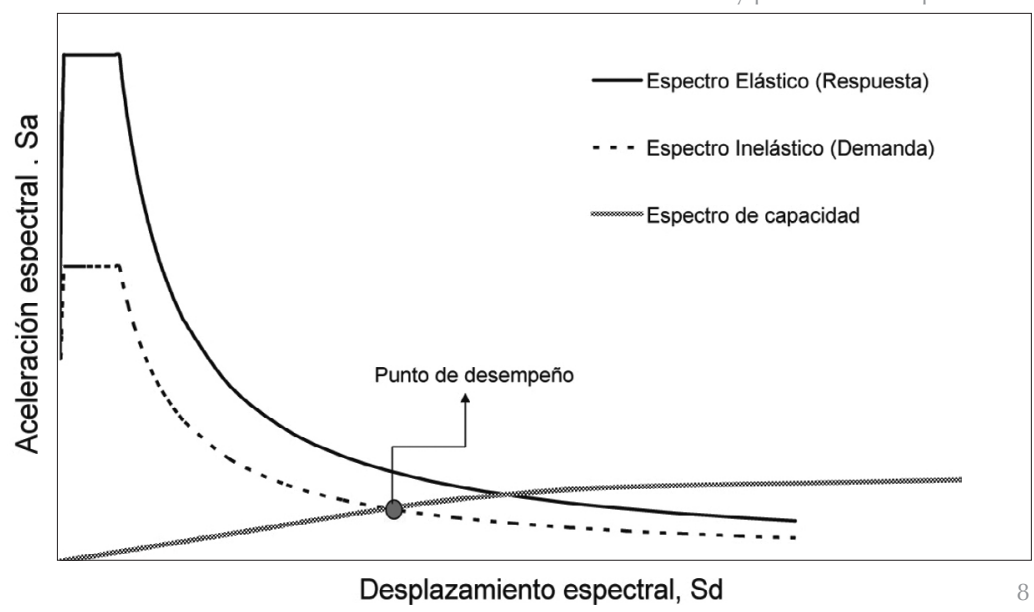


La Figura 7 y la Tabla 4 muestran la definición de los umbrales de los estados de daño en función de los puntos de capacidad de cedencia y capacidad última (13).

La estimación de la desviación estándar no es un problema trivial, en la metodología HAZUS (17) se definen para distintos tipos de tipologías, basándose en ensayos numéricos y en la opinión de expertos. Dentro del proyecto Risk-UE (2) se asume que el daño sísmico esperado sigue una distribución de probabilidad binomial y que la probabilidad de cada estado de daño, en su desplazamiento espectral medio, es del 50\% (18).

Finalmente, para cada escenario sísmico y para cada edificio se obtienen las matrices de probabilidad de daño, las cuales se obtienen a partir de las curvas de fragilidad y del punto de capacidad por demanda. Para esto, es necesario entrar en las curvas de fragilidad con el punto de desempeño y obtener las probabilidades correspondientes a cada estado de daño.

Para analizar el daño sísmico se han considerado 5 estados de daño: no daño, daño leve, daño moderado, severo y completo. El parámetro ponderado de daño medio que se suele usar para cuantificar los resultados se define como: [2]

$$
d_{M}=\sum_{i=0}^{i=4} E D_{i} \cdot P\left(E D_{i}\right)
$$

Donde $i$ corresponde a cada estado de daño considerado y $P\left(E D_{i}\right)$ es la probabilidad de ocurrencia del estado de daño $i$ dado un desplazamiento espectral. El índice $i$ toma valores de 0 , 1, 2, 3 y 4 para el estado de daño: no daño, leve, moderado, severo y completo, respectivamente.

Este parámetro de daño medio $\left(d_{M}\right)$ tiene intervalos de variación para cada estado de daño y, además, se puede utilizar para representar escenarios de riesgo sísmico en un área determinada. Para los cinco estados de daño considerados (no daño, leve, moderado, severo y completo) los intervalos de variación de $d_{M}$ son mostrados en la Tabla 5.

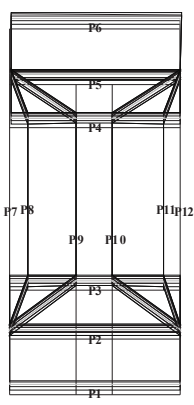

Casti

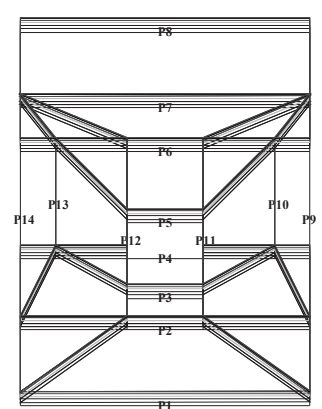

Pelai

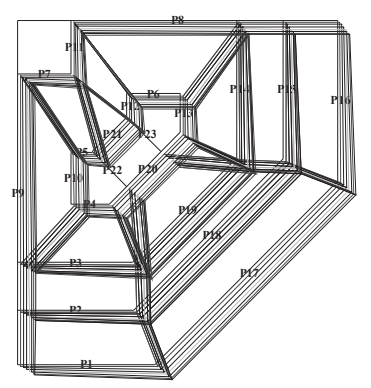

Angolo
Tabla 5

Intervalos de variación del parámetro de daño medio

\begin{tabular}{|c|c|}
\hline Umbrales de daño & Variación \\
\hline No daño & $0 \leq \mathrm{d}_{M}<0,5$ \\
\hline Leve & $0,5 \leq \mathrm{d}_{M}<1,5$ \\
\hline Moderado & $1,5 \leq \mathrm{d}_{M}<2,5$ \\
\hline Severo & $2,5 \leq \mathrm{d}_{M}<3,5$ \\
\hline Completo & $3,5 \leq \mathrm{d}_{M} \leq 4,0$ \\
\hline
\end{tabular}

\section{ANÁLISIS DE LA CAPACIDAD Y FRAGILIDAD ESTRUCTURAL DE LOS EDIFICIOS DE OBRA DE FÁBRICA}

En este apartado se evalúa la capacidad y fragilidad de los edificios de obra de fábrica de ladrillo, ampliamente, existentes en la ciudad de Barcelona. Después de describir las características de los edificios tipo aquí presentados y, definir, brevemente, la metodología a utilizar se ha realizado un análisis modal para obtener las propiedades dinámicas de cada edificio, así como también se ha realizado un análisis estático no lineal mediante el programa TreMuri.

Para cada modelo estructural se han obtenido sus propiedades modales. La Tabla 6 muestra las características modales correspondientes a los 3 primeros modos de vibración de los edificios Casti, Pelai y Angolo. A partir de un análisis modal se obtienen las propiedades dinámicas de cada estructura. En la Tabla 6, T es el período natural de vibración en segundos. Para la evaluación de cada edificio se ha elegido el modo de vibración que produce un desplazamiento longitudinal en dirección " $y$ ", perpendicular a la calle, es decir, se han utilizado las propiedades modales correspondientes al modo 3 para los edificios Casti y Pelai y al modo 1 para el edificio Angolo. La Figura 9 muestra el modo de vibración, en planta, para los 3 edificios, en la dirección principal del análisis.

A partir del análisis estático no lineal se obtienen las curvas de capacidad para cada uno de los edificios analizados, donde el patrón de fuerzas horizontales corresponde con el modo de vibración elegido. Seguidamente, cada curva de capacidad es transformada a espectro de capacidad para su posterior comparación con el espectro de demanda. La Figura 10 muestra las curvas de capacidad obtenidas para los edificios Casti, Pelai y Angolo. La Tabla 7 muestra los valores que definen la curva bilineal de los espectros de capacidad. 
Tabla 6

Valores modales para los edificios Casti, Pelai y Angolo

\begin{tabular}{|c|c|c|c|}
\hline Casti & Modo 1 (Despl. $x)$ & Modo 2 (Rot. $\theta$ ) & Modo 3 (Despl. y) \\
\hline $\mathrm{T}(\mathrm{s})$ & 0,85 & 0,66 & 0,56 \\
\hline$\%$ masa $x$ & 85 & 4 & 0 \\
\hline$\%$ masa y & 0 & 0 & 76 \\
\hline$\%$ masa z & 0 & 0 & 0 \\
\hline Pelai & Modo 1 (Despl. x) & Modo 2 (Rot. $\theta$ ) & Modo 3 (Despl. y) \\
\hline $\mathrm{T}(\mathrm{s})$ & 1,24 & 0,84 & 0,80 \\
\hline$\%$ masa $x$ & 91 & 2 & 0 \\
\hline$\%$ masa y & 0 & 0 & 83 \\
\hline$\%$ masa z & 0 & 0 & 0 \\
\hline Angolo & Modo 1 (Despl. y) & Modo 2 (Despl. x) & Modo 3 (Rot. $\theta$ ) \\
\hline $\mathrm{T}(\mathrm{s})$ & 0,83 & 0,81 & 0,49 \\
\hline$\%$ masa $x$ & 26 & 44 & 7 \\
\hline$\%$ masa y & 38 & 30 & 9 \\
\hline$\%$ masa z & 0 & 0 & 0 \\
\hline
\end{tabular}

10. Curvas de capacidad bilineal para los edificios Casti, Pelai y Angolo.
Tabla 7

Parámetros que definen la curva de capacidad bilineal

\begin{tabular}{|c|c|c|c|c|}
\hline \multirow{2}{*}{ Tipo } & \multicolumn{2}{|c|}{$\begin{array}{c}\text { Capacidad } \\
\text { de cedencia }\end{array}$} & \multicolumn{2}{c|}{$\begin{array}{c}\text { Capacidad } \\
\text { última }\end{array}$} \\
\cline { 2 - 5 } & $\mathrm{Dy}(\mathrm{m})$ & Ay $(\mathrm{g})$ & $\mathrm{Du}(\mathrm{m})$ & $\mathrm{Au}(\mathrm{g})$ \\
\hline Casti & 0,015 & 0,19 & 0,071 & 0,20 \\
\hline Pelai & 0,025 & 0,12 & 0,17 & 0,10 \\
\hline Angolo & 0,011 & 0,12 & 0,051 & 0,12 \\
\hline
\end{tabular}

A partir de los espectros de capacidad bilineales, para cada edificio, se definen los desplazamientos espectrales medios para cada umbral del estado de daño para, a partir de aquí, desarrollar las curvas de fragilidad para cada edificio. La Tabla 8 muestra los parámetros que definen las curvas de fragilidad correspondientes a los edificios analizados. Las Figuras 11, 12 y 13 muestran las, respectivas, curvas de fragilidad para los edificios Casti, Pelai y Angolo.

\section{EVALUACIÓN DEL RIESGO SÍSMICO}

En este apartado se definen los escenarios sísmicos para estimar las matrices de probabilidad de daño (MPD) de cada uno de los edificios estudiados.

\subsection{Demanda sísmica}

La acción sísmica se considera en términos espectrales. Se considera un nivel de aceleración pico máxima (PGA) de 0,04g. Este valor ha sido tomado de acuerdo a la norma sísmica española vigente NCSE-02 (4) y corresponde a un período de retorno de 500 años. Para las formas de los espectros elásticos de respuesta con 5\% de amortiguamiento se han tomado los definidos en el Eurocódigo 8 (5), donde se consideran hasta 5 tipos de suelo: A, B, C, D y $E$, que van desde suelos duros (tipo $A$ ) a suelos

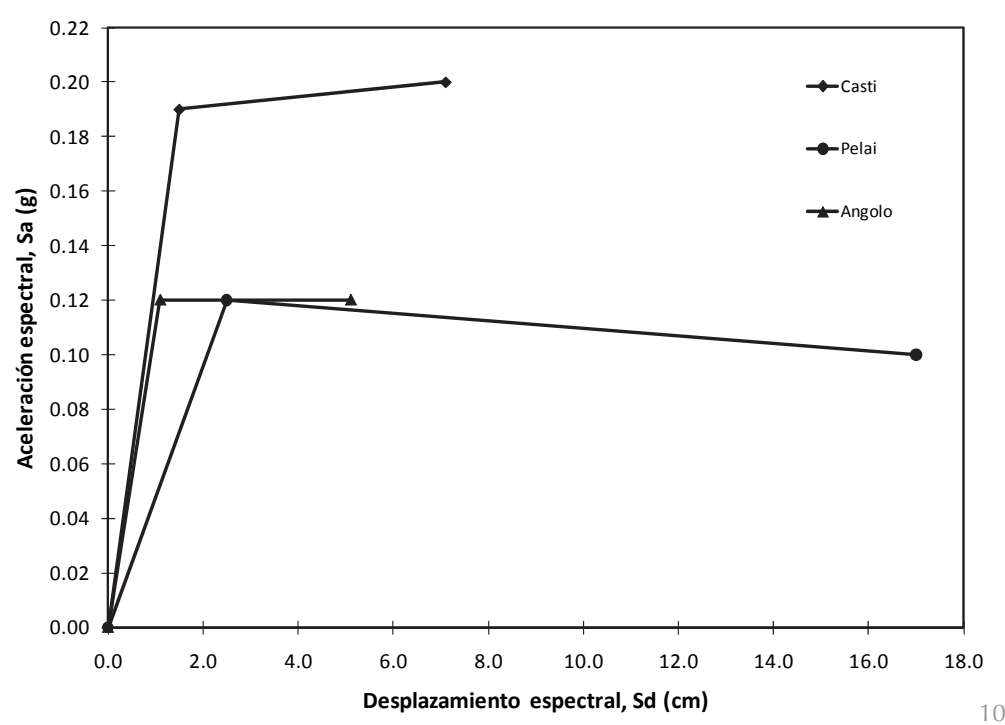

Tabla 8

Parámetros que definen las curvas de fragilidad, desplazamiento espectral medio y su variabilidad

\begin{tabular}{|l|c|c|c|c|c|c|c|c|}
\hline & $\overline{S d}_{1}(\mathrm{~cm})$ & $\beta_{1}$ & $\overline{S d}_{2}(\mathrm{~cm})$ & $\beta_{2}$ & $\overline{S d}_{3}(\mathrm{~cm})$ & $\beta_{3}$ & $\overline{S d}_{4}(\mathrm{~cm})$ & $\beta_{4}$ \\
\hline Casti & 1,07 & 0,99 & 1,53 & 0,97 & 2,93 & 0,90 & 7,12 & 0,88 \\
\hline Pelai & 1,77 & 0,99 & 2,53 & 0,97 & 6,09 & 0,90 & 16,76 & 0,88 \\
\hline Angolo & 0,77 & 0,99 & 1,11 & 0,97 & 2,10 & 0,90 & 5,09 & 0,88 \\
\hline
\end{tabular}

blandos (tipo D); los suelos tipo E corresponden a una configuración de suelos con un nivel aluvial de tipo C ó D sobre un material más duro (tipo A). De esta forma las características de los suelos tipo A, B, C y D del Eurocódigo 8 son comparables con los de tipo I, II, III y IV de la NCSE-02, si bien no hay una coincidencia total.

La Tabla 9 muestra los parámetros que definen los espectros de respuesta aquí utilizados. La Figura 14 muestra los espectros elásticos de respuesta para un amortiguamiento del 5\%. En la Tabla 9, S es un parámetro del suelo, $k_{1}$ y $k_{2}$ son los exponentes que definen la forma del espectro para un período de 

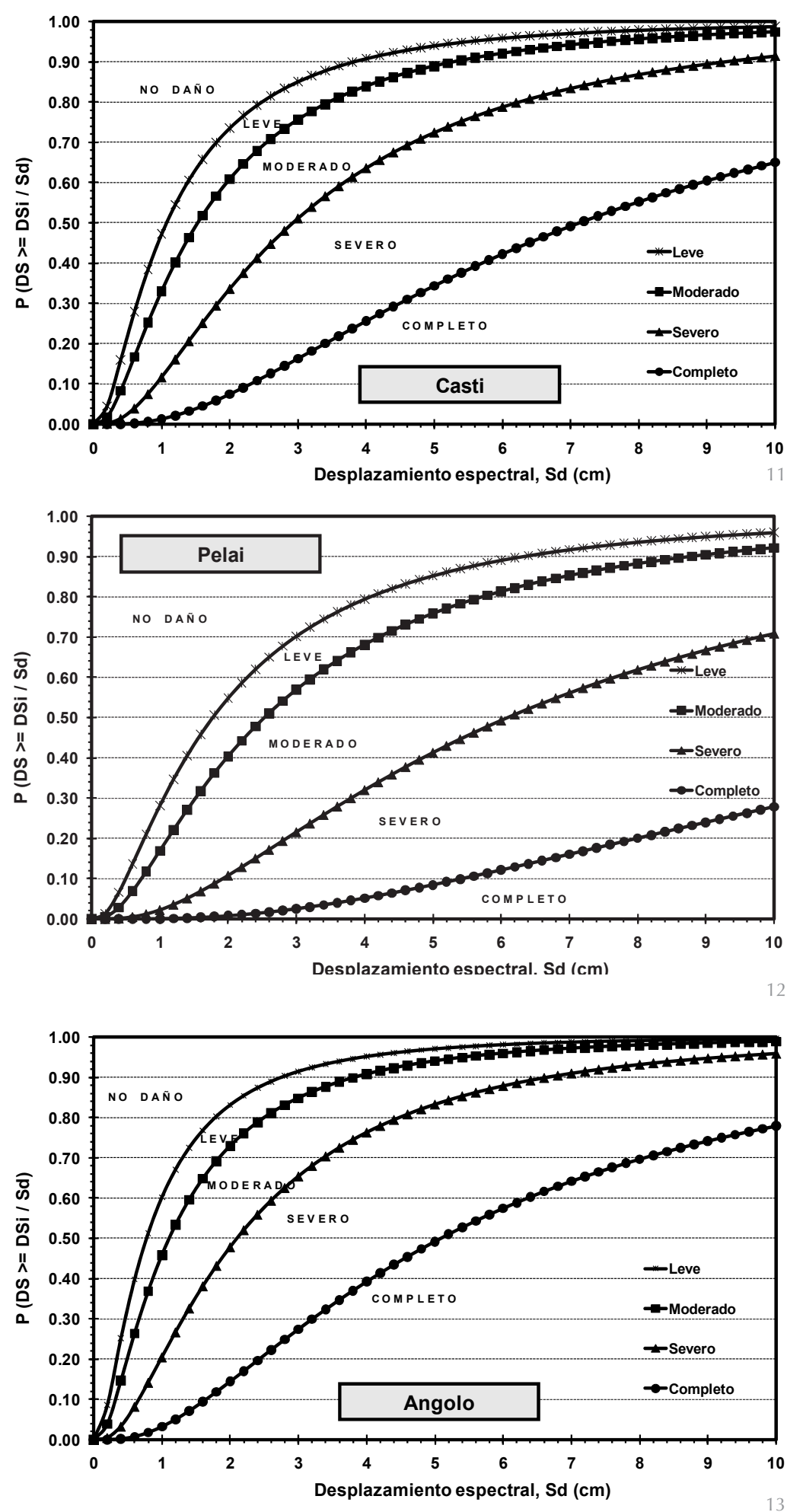

Tabla 9

Parámetros que definen

los espectros de respuesta

\begin{tabular}{|c|c|c|c|c|c|c|}
\hline $\begin{array}{c}\text { Tipo de } \\
\text { suelo }\end{array}$ & $\boldsymbol{S}$ & $\boldsymbol{k}_{\boldsymbol{1}}$ & $\boldsymbol{k}_{\mathbf{2}}$ & $\boldsymbol{T}_{\boldsymbol{B}}(\mathbf{s})$ & $\boldsymbol{T}_{\boldsymbol{C}}(\mathbf{s})$ & $\begin{array}{c}\boldsymbol{T}_{\boldsymbol{D}} \\
(\mathbf{s})\end{array}$ \\
\hline A & 1,00 & 1,0 & 2,0 & 0,15 & 0,40 & 2,0 \\
\hline B & 1,20 & 1,0 & 2,0 & 0,15 & 0,50 & 2,0 \\
\hline C & 1,15 & 1,0 & 2,0 & 0,20 & 0,60 & 2,0 \\
\hline D & 1,35 & 1,0 & 2,0 & 0,20 & 0,80 & 2,0 \\
\hline E & 1,40 & 1,0 & 2,0 & 0,15 & 0,50 & 2,0 \\
\hline
\end{tabular}

vibración mayor que $T_{C}$ y $T_{D}$, respectivamente. $T_{B}$ y $T_{C}$ son los límites del tramo de aceleración espectral constante y $T_{D}$ define el comienzo del tramo de desplazamiento constante del espectro (5).

\subsection{Respuesta sísmica}

A partir de los espectros de respuesta y de los espectros de capacidad, existen diversos procedimientos para estimar la respuesta sísmica, en este caso, representada por el desplazamiento espectral solicitado por un terremoto. En este trabajo se ha seguido el procedimiento indicado en el EC-8 (5), este procedimiento se halla perfectamente detallado en el Anexo B de dicho código. Se ha comprobado que los resultados obtenidos apenas difieren de los que se obtienen usando el procedimiento sugerido en el ATC-40 (16).

La Tabla 10 muestra los puntos de desempeño (puntos de capacidad por demanda) obtenidos para el escenario sísmico considerado. Se han tenido en cuenta los 5 tipos de suelos previstos en el EC-8.

Tabla 10

Puntos de capacidad por demanda.

\begin{tabular}{|c|c|c|c|c|c|}
\hline \multicolumn{5}{|c|}{ Puntos de desempeño, $\mathrm{Sd}_{\mathrm{PD}}(\mathrm{cm})$} \\
\hline Suelo & Suelo A & Suelo B & Suelo C & Suelo D & Suelo E \\
\hline Casti & 0,56 & 0,84 & 1,03 & 2,15 & 0,98 \\
\hline Pelai & 0,93 & 1,39 & 1,60 & 2,50 & 1,62 \\
\hline Angolo & 0,61 & 0,91 & 1,05 & 2,15 & 1,07 \\
\hline
\end{tabular}

\subsection{Matrices de probabilidad de daño}

Entrando en las curvas de fragilidad con el punto de capacidad por demanda se han obtenido las matrices de probabilidad de daño. La Tabla 11 muestra las MPD para el escenario sísmico considerado para los edificios típicos de Barcelona.

\section{DAÑO ESPERADO}

El grado de daño medio indica el estado o grado de daño que tiene una mayor probabilidad de ocurrencia. La Tabla 12 proporciona los factores de daño medio obtenidos a partir de las matrices de probabilidad de daño para el escenario sísmico considerado y para cada edificio.

\section{DISCUSIÓN Y CONCLUSIÓN}

El tipo de suelo $E$ es un suelo formado por un sustrato duro (tipo A o B) sobre el que se halla un terreno blando (tipo D) de considerable espesor. Se observa cómo los valores del factor de daño medio para este tipo de suelo, Tabla 12, son similares a los valores obtenidos para suelos tipo B o tipo C; por lo tanto, la discusión se va a centrar en los suelos tipos A, B, C y D que van de mejor (tipo A) a peor calidad (tipo D). 
Tabla 11

Matrices de probabilidad de daño, valores en \%

\begin{tabular}{|c|c|c|c|c|c|}
\hline & $\begin{array}{c}0 \text { No } \\
\text { daño }\end{array}$ & $\begin{array}{c}1 \\
\text { Leve }\end{array}$ & $\begin{array}{c}2 \text { Mode- } \\
\text { rado }\end{array}$ & $\begin{array}{c}3 \\
\text { Severo }\end{array}$ & $\begin{array}{c}4 \\
\text { Completo }\end{array}$ \\
\hline & \multicolumn{5}{|c|}{ Suelo A } \\
\hline Casti & 74 & 11 & 12 & 3 & 0 \\
\hline Pelai & 75 & 10 & 13 & 2 & 0 \\
\hline Angolo & 60 & 13 & 19 & 7 & 1 \\
\hline & \multicolumn{5}{|c|}{ Suelo B } \\
\hline Casti & 60 & 13 & 19 & 7 & 1 \\
\hline Pelai & 60 & 14 & 21 & 5 & 0 \\
\hline Angolo & 43 & 15 & 24 & 16 & 2 \\
\hline & \multicolumn{5}{|c|}{ Suelo C } \\
\hline Casti & 52 & 14 & 22 & 11 & 1 \\
\hline Pelai & 54 & 16 & 23 & 7 & 0 \\
\hline Angolo & 38 & 15 & 25 & 18 & 4 \\
\hline & \multicolumn{7}{|c|}{ Suelo D } \\
\hline Casti & 24 & 13 & 27 & 28 & 8 \\
\hline Pelai & 36 & 14 & 34 & 14 & 2 \\
\hline Angolo & 15 & 10 & 24 & 35 & 16 \\
\hline & \multicolumn{7}{|c|}{ Suelo E } \\
\hline Casti & 54 & 14 & 21 & 10 & 1 \\
\hline Pelai & 54 & 14 & 25 & 6 & 1 \\
\hline Angolo & 37 & 14 & 26 & 19 & 4 \\
\hline
\end{tabular}

Tabla 12

Grado de daño medio esperado

\begin{tabular}{|c|c|c|c|c|c|}
\hline \multicolumn{7}{|c|}{$\boldsymbol{d}_{\boldsymbol{M}}$} \\
\hline & Suelo A & Suelo B & Suelo C & Suelo D & Suelo E \\
\hline Casti & 0,44 & 0,76 & 0,95 & 1,83 & 0,90 \\
\hline Pelai & $\mathbf{0 , 4 2}$ & 0,71 & 0,83 & 1,32 & 0,86 \\
\hline Angolo & 0,76 & 1,19 & 1,30 & $\mathbf{2 , 2 7}$ & 1,39 \\
\hline
\end{tabular}

La Tabla 11 muestra, para cada edificio y para cada emplazamiento, la probabilidad que se dé un estado de daño. En ella se observa como para los 3 edificios analizados la probabilidad del estado de no daño disminuye considerablemente de un suelo tipo A hasta un suelo tipo D; para el estado de daño leve, prácticamente, los valores se mantienen iguales; a partir del estado de daño moderado las probabilidades aumentan cuando se va de un suelo tipo $\mathrm{A}$ a un suelo tipo $\mathrm{D}$, siendo considerable y gradual el aumento en el estado de daño severo; mientras que para el estado de daño completo el aumento es importante al situarse las estructuras en un suelo tipo D.

Observando la Tabla 12, para el escenario sísmico considerado, el menor daño esperado se obtiene para el edificio Pelai situado en un suelo tipo A. El mayor daño esperado corresponde al edificio Angolo situado en un suelo tipo D. Este comportamiento se mantiene para todos los tipos de suelo. Este buen y mal comportamiento sísmico se debe a la mayor regularidad del edificio Pelai y a la mayor irregularidad del edificio Angolo. También contribuyen a la mayor fragilidad del edificio Angolo la disposición de muros y paredes que configuran su sistema resistente.
En general, para los 3 edificios el daño aumenta al disminuir la calidad del suelo. El aumento del daño esperado es espectacular para el edificio Angolo, que pasa de un estado de daño leve $(0,76)$ cuando se halla en suelo duro (tipo A) a un estado de daño moderado $(2,27)$ cuando se halla en suelos blandos (tipo D). De los 3 edificios, Angolo es el que muestra el peor comportamiento sísmico y Pelai el mejor. Ello confirma los beneficios de la regularidad geométrica y de distribución de muros de los edificios que queda patente también en todos los códigos sismo-resistentes. La irregularidad geométrica, además, dificulta la disposición adecuada de los elementos estructurales resistentes, lo que debilita aún más el edificio. En un suelo tipo $\mathrm{D}$, los grados de daño esperados para los 3 edificios son, respectivamente, 1,83, 1,32 y 2,27 , de forma que la introducción de una mayor regularidad en planta puede llegar a disminuir el daño esperado en casi un grado.

Finalmente, en la Tabla 12 se observa que el daño esperado es alto teniendo en cuenta el escenario sísmico considerado ( $\mathrm{PGA}=0,04 \mathrm{~g}$ ) que de acuerdo a la norma sísmica, corresponde a una intensidad básica EMS=VI. Para el edificio Angolo situado en un suelo tipo $\mathrm{D}$ (suelo blando) el factor de daño medio es 2,27 , para este factor se tiene una probabilidad del estado de daño severo del 35\% y una probabilidad del $16 \%$ de "colapso".

Si se considera que la amplificación del suelo blando incrementa en un grado la intensidad macrosísmica básica, se concluye que, para una intensidad VII, muchos edificios tipo Angolo sufren daños severos y muchos sufren colapso, por lo que se debería incluir este tipo de edificios dentro de la clase más vulnerable (clase de vulnerabilidad A) de las cinco clases de vulnerabilidad previstas en la escala EMS98 (19). Por otra parte, el edificio Pelai situado en suelo tipo A, sufre un grado de daño medio de 0,42 y las probabilidades de los estados de daño son del $10 \%$ para el estado de daño leve y del $13 \%$ para el estado de daño moderado. Es decir, para una intensidad VI, varios edificios sufren daños moderados. Teniendo en cuenta que las probabilidades del $10 \%$ y del $13 \%$ se hallan en la frontera entre las cantidades consideradas como pocos y muchos en la escala EMS-98 y con la descripción del grado de daño $\mathrm{VI}$, se concluye que los edificios tipo Pelai se encuentran dentro de la clase de vulnerabilidad B.

Los estudios de vulnerabilidad sísmica son significativos para la predicción y prevención del daño sísmico esperado. La metodología aquí empleada se basa en el análisis estático no lineal y en el método del espectro de capacidad. De particular interés es la aplicación de este tipo de técnicas en zonas de sismi- 
cidad moderada a baja, donde el desarrollo de las ciudades y de los entornos urbanos ha significado un aumento del riesgo sísmico. La metodología aquí utilizada también ha sido aplicada en edificios de hormigón armado (15) y en edificios metálicos (20).

Los modelos utilizados en el análisis corresponden a edificios reales, existentes en el distrito Eixample de Barcelona. Las propiedades mecánicas empleadas para la mampostería son típicas de Barcelona, por lo tanto, se puede considerar que las matrices de probabilidad de daño y los valores de daño medio obtenidos son razonables de esperar para el escenario considerado.

Finalmente, se concluye que los edificios de obra de fábrica de ladrillo de Barcelona muestran una alta vulnerabilidad que puede clasificarse entre la clase A y la clase B de las clases de vulnerabilidad tipificadas en la escala EMS-98 (19).

\section{AGRADECIMIENTOS}

Este trabajo estuvo parcialmente financiado por el proyecto de investigación: Desarrollo y aplicación de procedimientos avanzados para la obtención de escenarios de riesgo sísmico: REN2001-2418-C04-01 RIES, Ministerio de Ciencia y Tecnología. Por una beca Marie Curie Fellowships Association. Además queremos agradecer al Ing. Vicente Alegre por su ayuda incondicional, así como también, la primera autora agradece a los profesores Sergio Lagomarsino, Ángel Aparicio y Luis Pujades.

\section{BIBLIOGRAFÍA}

(1) Infocca: "Funcions d'informació de Barcelona i Cartografia". Informe interno, Institut Municipal d'Informàtica (IMI), Ayuntamiento de Barcelona, (1992).

(2) Milutinovic Z. V.; Trendafiloski G. S.: "Vulnerability of current buildings. WP-4 Report". Work Package 4 of Risk-UE Project, European Commission, EVK4-CT-2000-00014. (2003).

(3) Moreno R.; Lantada N.; Bonett R.; Pujades L. and Barbat A.: "WP4: Vulnerability assessment of current buildings. Capacity and fragility of the Barcelona's residential buildings". Report by CIMNE Working Group to Risk-UE project, Barcelona, (2003).

(4) NCSE-02: Normativa "Norma de Construcción Sismorresistente: Parte General y Edificación", Real Decreto 997/2002, Boletín Oficial del Estado: 244, Madrid, (2002).

(5) EC-8: Eurocode 8: "Design of structures for earthquake resistance - Part 1: General rules, seismic actions and rules for buildings". Comité Européen de Normalisation. EN 1998-1:2004, CEN Brussels, December, (2004), 229 pp.

(6) Galasco, A.; Lagomarsino, S. ; Penna, A.: "TREMURI Program: Seismic Analyzer of 3D Masonry Buildings". Technical Report, Universitat degli Studi di Genova, Italia, (2002).

(7) Cerdà, I.: Teoría General de la Urbanización. Reforma y Ensanche de Barcelona. Reedición: Instituto de estudios fiscales, Barcelona, (1968).

(8) García Espuche, A.: El quadrat d'or. Centre de la Barcelona modernista. La formació d'un espai urbà privilegiat. Olimpiada cultural Barcelona'92. Lunwerg Editores, 315 pp. (1990).

(9) Paricio, A.: Secrects d'un sistema constructiu: I'Eixample. Universidad Politécnica de Cataluña. Ediciones UPC, Barcelona, (2001).

(10) Marí, A.; Alegre, V.; Gens, A.; Roca, P. et al.: Estudio preliminar sobre los posibles efectos de la construcción de un túnel para el tren de alta velocidad bajo los edificios situados entre las calles Mallorca, Valencia, Lepanto y Cartagena. Universidad Politécnica de Cataluña, España, (2003).

(11) Gambarotta, L.; Lagomarsino, S.: "Damage model for the seismic response of brick masonry shear walls. Part II: the continuum model and its applications". Earthquake Engineering and Structural Dynamics, 26, (1997), pp. 441-462

(12) Penna, A.: "A macro-element procedure for the non-linear dynamic analysis of masonry buildings". Tesis Doctoral, Politecnico di Milano, Italia, (2002).

(13) Galasco, A.; Lagomarsino, S.; Penna, A.; Resemini, S.: "Non-linear seismic analysis of masonry buildings". $13^{\text {th }}$ World Conference on Earthquake Engineering, paper 843, Vancouver, Canadá, (2004).

(14) Yépez, F.: "Metodología para la evaluación de la vulnerabilidad y riesgo sísmico de estructuras aplicando técnicas de simulación". Tesis Doctoral. Universidad Politécnica de Cataluña, España, (1996).

(15) Moreno, R.: "Evaluación del riesgo sísmico en edificios mediante análisis estático no lineal: Aplicación a diversos escenarios sísmicos de Barcelona". Tesis Doctoral. Universidad Politécnica de Cataluña, Barcelona, España, (2006).

(16) ATC-40: "Seismic Evaluation and Retrofit of Concrete Buildings". Applied Technology Council, Report: SSC 96-01, volume 1, Seismic Safety Commission, Redwood City, California, (1996).

(17) HAZUS 99-SR2: "HAZUS Technical Manual". Federal Emergency Management Agency, FEMA \& National Institute of Building Sciences, NIBS, Washington D.C.; vols. 1, 2, 3, (2002).

(18) Barbat, A. H.; Pujades, L.G.; Lantada N.; Moreno R.: "Seismic damage evaluation in urban areas using a capacity spectrum based method: Application to Barcelona." Soil Dynamics and Earthquake Engineering, 28, 10-11, (2008), pp. 851-865. Erratum:Volume 30, 8 (2010), pp. 767. doi: 10.1016/j.soildyn.2009.12.014.

(19) Grünthal, G.: "European Macroseismic Scale 1998". Centre Européen de Géodynamique et Séismologie, Cahiers du Centre Européen de Géodynamique et de Séismologie, Volume 15, Luxemburg, (1998).

(20) Moreno González, R.; Bairán García, J.M. y Rodas Aldana, W.A.: "Vulnerabilidad sísmica en edificios metálicos". Hormigón y Acero, 62, 261, 69-80, (2011). 